\title{
La Terapia Ocupacional en Colombia prosigue su avance, entre aspiraciones y logros
}

\author{
Occupational Therapy Growth in Colombia: \\ Purposes and Achievements
}

Alicia Trujillo Rojas ${ }^{1}$

Recibido: Junio 112012 • Aceptado: Junio 132012

Trujillo, A. (2013). La Terapia Ocupacional en Colombia prosigue su avance, entre aspiraciones y logros. Revista Ocupación Humana, 13 (1), pp. 5-18.

Resumen: Este artículo presenta un panorama amplio del crecimiento de la Terapia Ocupacional en Colombia, desde la postura teórica de su desarrollo como profesión. Atiende en particular a los logros alcanzados en el fortalecimiento del conocimiento; la formación académica; la especificidad y cobertura de los servicios humanos; y la presencia asociativa. A manera de conclusiones y recomendaciones, el segmento final reflexiona sobre el futuro, en lo que se refiere a algunos aspectos del fondo de saber específico; la ocupación humana como directriz fundamental; la capacidad asociativa; y las cualidades protagónicas que deben demostrar los terapeutas ocupacionales.

Palabras clave: Profesión, Campo de conocimiento, Ocupación humana, Servicios humanos, Agremiación.

Abstract: This article examines the development of occupational therapy in Colombia from the sociological perspective of how professions originate and evolve. The objective is to determine advancement and to identify potencial for progress in four dimensions: body of knowledge; human occupation as the fundamental and specific domain of study; professional association; and profile of occupational therapists as agents of professional improvement.

Key words: Profession, Body of knowledge, Human occupation, Human services, Association.

\section{Introducción}

Nos encontramos ante una doble y muy importante celebración: el cuadragésimo aniversario de la Fundación de la Asociación Colombiana de Terapia Ocupacional (ACTO), recientemente transformada en el Colegio Colombiano de Terapia Ocupacional (CCTO) y el regreso a la circulación de la Revista Ocupación Humana, tribuna por excelencia para socializar resultados investigativos y posturas conceptuales y experienciales de los terapeutas ocupacionales. Se trata de dos hechos significativos que indican la fortaleza acumulada durante cuatro décadas y por lo cual congratulamos a la Junta Directiva, a la Asamblea General y a toda la comunidad profesional.

\footnotetext{
${ }^{1}$ Terapeuta Ocupacional (OTR). Master of Arts en Construcción Teórica. Profesora Emérita, Universidad Nacional de Colombia. alitrujillo2003@yahoo.com
} 
Con esos motivos, se nos ha pedido reflexionar sobre la trayectoria de la Terapia Ocupacional en Colombia. Para ello adoptaremos la perspectiva de mirar sus características genéricas como profesión, a la manera planteada por la literatura sociológica que estudia el surgimiento y desarrollo de las profesiones. En primer lugar se formulará lo que aquí se denomina "una aspiración" o propósito amplio de la profesión. Con base en esa visión se hará referencia a los logros y a las dificultades detectadas. Finalmente se propondrán unas direcciones hacia el futuro, a manera de temas para debatir durante la construcción de planes y proyectos de evaluación o desarrollo educativo, asociativo y de programación en servicios humanos.

El presente aporte es una postura personal, asociada con la trayectoria de vida de la autora: en programas de Terapia Ocupacional infantil y de salud mental; durante su experiencia académica en gestión universitaria, docencia de pregrado y postgrado e investigación en Terapia Ocupacional, ciencia de la ocupación humana y discapacidad e inclusión social; y como impulsora y representante de la Asociación Colombiana de Terapia Ocupacional en diversos momentos.

\section{Aspiración para Terapia Ocupacional}

Los estudios sociológicos afirman que las profesiones surgen a partir de grupos ocupacionales que crecen y se sofistican como consecuencia de su número de vinculados, la instauración de un proceso educativo formal, el fondo de saber específico que construyen, el poder organizativo que demuestran, los mecanismos de regulación que emplean incluyendo estándares profesionales y normas éticas (Etzioni, 1969) y la utilidad de los servicios que ofrecen a la sociedad. Desde el siglo XIX ese ha sido el proceso seguido por la Terapia Ocupacional en países como Canadá, Estados Unidos y Gran Bretaña, entre otros.

En Colombia se cumple una historia similar (Trujillo, 2002, pp. 389465). Es así como una gran aspiración de la colectividad de Terapia Ocupacional ha sido -desde su fundación en 1966- la de avanzar en solidez profesional con identidad, sustentada en un proceso educativo de nivel universitario básico y postgraduado, que permita a sus representantes: a) la adquisición, demostración y actualización de credenciales intelectuales y comunicativos amplios, profesionales específicos y de crecimiento personal y ciudadano; y b) una creciente capacidad de agremiación y autorregulación del ejercicio profesional, todo ello para bienestar del grupo profesional, de las poblaciones beneficiarias de sus servicios y de la sociedad.

Sobre el tema de la profesionalización o nivel profesional de la Terapia Ocupacional en Colombia se ha reflexionado de manera directa o indirecta en muchos eventos de la ACTO, tanto en debates de su Junta Directiva; como en ponencias de Congresos nacionales e internacionales; en la Asociación Colombiana de Facultades de Terapia Ocupacional (ACOLFAC$\mathrm{TO})$; en foros internacionales (Confederación Latinoamericana de Terapia Ocupacional (CLATO); en libros y otras publicaciones; y en artículos de la Revista Ocupación Humana. También ha sido materia de atención en 
las Universidades que ofrecen programas curriculares de Terapia Ocupacional, durante los cuales se adquiere un conjunto de saberes amplios y especializados, contextualizados en la realidad socio política amplia. A mayor fortaleza de una profesión, mayor probabilidad de ejercer autonomía ${ }^{2}$, entendida aquí como la capacidad de autodeterminación, es decir adoptar decisiones de manera independiente y ejercer control sobre el propio ejercicio profesional (Wikipedia, 2011) con argumentos juiciosos.

La autonomía, sin embargo, no está exenta de condiciones. Al respecto Sánchez y Sáez (2012) indican que la autodeterminación sólo puede perdurar si va acompañada de mecanismos constantes de autoevaluación y regulación de procesos y comportamientos. En el caso de la Terapia Ocupacional, dichos mecanismos deben ser puestos en marcha por los centros educativos, los prestadores de servicios y las organizaciones profesionales. Algunas medidas empleadas son el diseño de estándares de actuación profesional, la evaluación por pares, el establecimiento de principios y normas éticas y otros procedimientos que permitan orientar y vigilar el cumplimiento de la normatividad establecida y aseguren un monitoreo permanente del desempeño profesional como conjunto. Una actuación profesional basada en conocimiento y experticia, reconocida por su carácter singular, acogida a estándares y suministrada de manera experta, oportuna y efectiva a las personas y grupos sociales que lo requieren genera reconocimiento y prestigio en el ámbito social.

Asegurar el avance profesional de la Terapia Ocupacional en Colombia exige la contribución permanente de un conjunto amplio de agentes que asuman responsabilidades en los ámbitos académico, asociativo/gremial y de servicios humanos. Dada la multiplicidad de tareas que se deben cumplir, es deseable que un número creciente de terapeutas ocupacionales participe simultáneamente en los tres ámbitos o por lo menos en dos de ellos, siempre y cuando la vinculación a la agremiación más representativa, el CCTO, sea una constante. En los países donde la Terapia Ocupacional es una profesión con claro posicionamiento social, una amplia mayoría de sus integrantes está vinculada a la agremiación profesional e impulsan diálogos permanentes entre la academia, los servicios humanos y la asociación profesional. Esto se debe a que el fortalecimiento continuado de la base de conocimientos; la formación profesional específica; la cobertura, pertinencia social y oportunidad de los servicios humanos; la investigación coherente con necesidades sociales y profesionales; el monitoreo de estándares, procedimientos de actuación y regulación ética; y la participación e intercambio con diversos organismos y actores sociales,

\footnotetext{
${ }^{2}$ La autonomía no debe concebirse en términos absolutos sino interconectados. Las profesiones, como las personas, son parte y consecuencia de la totalidad local, nacional, regional y global. En esa medida están influenciadas por y vinculadas a ordenamientos sociales amplios y a dinámicas y fuerzas externas que establecen controles políticos y sociales y ejercen poder directo e indirecto. Las organizaciones profesionales han de reconocer esas fuerzas, entenderlas y establecer interlocuciones tendientes a hacer presencia en los contextos extraprofesionales, armonizar esfuerzos, resolver diferencias, encontrar convergencias y solucionar conflictos en beneficio de la profesión y de la sociedad como conjunto.
} 
nacionales e internacionales, constituye una empresa de gran envergadura que obliga a la reflexión y planeación conjunta de expertos con experiencias variadas y a la generación de alianzas de diverso nivel. Además, para afrontar exitosamente las complejidades de la sociedad global y poder evolucionar y actualizarse de acuerdo al ritmo acelerado del mundo tecnológico, económico, político, cultural y de información, es altamente conveniente establecer vínculos e intercambios entre los diversos estamentos de la profesión y con otros saberes y representantes de la sociedad.

\section{Avances}

La pregunta que surge es: ¿cuáles son los logros alcanzados por la Terapia Ocupacional colombiana hacia la aspiración expuesta en la sección precedente? En términos generales puede responderse que es mucho el terreno ganado en relación con el comienzo de la profesión en 1966 y con la fundación de la ACTO en 1972. Los avances deben entenderse dentro de la perspectiva de un proceso, en el cual surgen dificultades que o se sobrepasan o se superan parcialmente, para seguir adelante hacia metas mayores. En esta presentación se privilegian solamente algunos aspectos de los criterios señalados en los estudios sociológicos de las profesiones: fondo de conocimientos específico; formación académica; cobertura, utilidad y pertinencia de los servicios para la sociedad; y presencia y gestión gremial.
Una identificación más amplia y profunda de aspectos a analizar puede alcanzarse al consultar la Revista Ocupación Humana (ROH) (1984-2006), las Memorias de los quince Congresos profesionales patrocinados por la ACTO $^{3,4}$, textos que abarcan desde los antecedentes e iniciación en el siglo XX hasta la realidad vigente durante los primeros años del siglo XXI (TrujiIlo, 2002) o publicaciones que cubren áreas determinadas del ejercicio de la Terapia Ocupacional (Álvarez, 2010; Villamil, Mojica y Puerto, 2006). Por ejemplo, sería muy útil precisar logros, en extensión y profundidad, por medio de estudios longitudinales o de análisis de contenido que exploren características y temáticas en publicaciones seriadas de la última década y en el Catálogo Latinoamericano de Libros y Revistas de Terapia Ocupacional (Palm y cols., 2012).

\section{Fondo de conocimiento}

La profesión posee un saber específico creciente referente al desempeño ocupacional de los seres humanos en situaciones de bienestar y ante la evidencia de trastornos e interrupciones de la actuación ocupacional a lo largo del ciclo vital y para la recuperación de la calidad de vida. Se dispone de marcos conceptuales, posturas teóricas, metodologías y procedimientos de evaluación e intervención, los cuales han mostrado un auge en países anglosajones, a partir de la década de 1980. Cuando se estima pertinente, el saber propio de la Terapia Ocupacional se

\footnotetext{
${ }^{3}$ El 16을 Congreso Colombiano de Terapia Ocupacional tendrá lugar en Bogotá en el mes de Septiembre de 2012.

${ }^{4}$ Nota de la editora: Este artículo fue escrito por la autora y aceptado para publicación en la Revista Ocupación Humana en 2012, previo a la cancelación del 16으 Congreso Colombiano de Terapia Ocupacional.
} 
correlaciona con fondos de información o enfoques provenientes de disciplinas y profesiones relacionadas.

Un volumen alto del conocimiento existente en Colombia proviene de fuentes internacionales, debido a tres debilidades: insuficiente investigación, tanto como divulgación de resultados y sistematización de experiencias y baja representación de formación postgraduada en la hoja de vida de los miembros de la comunidad de Terapia Ocupacional (aproximadamente $29 \%$ a comienzos del actual milenio, Trujillo 2002, p. 524). Si bien este porcentaje supuestamente se ha venido incrementando en los últimos años, aún no se dispone de estadísticas globales que permitan determinar su magnitud. Recordemos que la educación postgraduada es el ámbito más propicio para la formación investigativa y para el surgimiento y socialización de propuestas que complementen, profundicen o transformen el conocimiento disponible; así como los resultados investigativos contribuyen a incrementar el bagaje de conocimiento $y$, por esa vía, fortalecen la identidad de la profesión.

\section{Formación académica}

Los diez programas de Terapia Ocupacional vigentes en el país (Palm y cols., 2012) son de nivel universitario básico. Para ellos existen, desde finales del siglo XX y comienzos del XXI, dos hechos importantes que contribuyen a garantizar la calidad de la formación: el Sistema Nacional de Acreditación $(\mathrm{SNA})^{5}$ y las pruebas de Estado lideradas por el Instituto Colombiano de la Educación Superior (ICFES), antes denominadas Examenes de Calidad de la Educación Superior (ECAES), ahora llamadas Pruebas PRO (ECAES) ${ }^{6}$.

Es indudable que tanto la autoevaluación de los programas académicos como la evaluación aplicada a estudiantes de último año son instrumentos potentes para promover el mejoramiento de los planes curriculares y toda su estructura. En el caso de Terapia Ocupacional, la experiencia ha contribuido a movilizar recursos académicos y de gestión administrativa hacia niveles más altos y ha propiciado la cultura del mejoramiento de la calidad y el compartir hallazgos y planear estrategias de superación entre univer

\footnotetext{
${ }^{5}$ En Colombia, con base en el Artículo 53 de la Ley 30 de 1992, todos los programas universitarios hacen parte del sistema de educación superior y como tal son instados a transitar hacia el cumplimiento de la acreditación de la calidad, a partir de un proceso de autoevaluación y de evaluación por pares, que se inició en 1998, en el cual intervienen la Institución evaluada, la comunidad académica y el Consejo Nacional de Acreditación (CNA) como instancia del Estado colombiano. Las políticas, estrategias y procesos que componen el SNA son importante en la medida que buscan, a través de la autoevaluación y la evaluación por pares académicos, promover altos requisitos de calidad en los integrantes del sistema. (Google, Sistema Nacional de Acreditación, Colombia, Mayo 20 de 2012).

${ }^{6}$ Los ECAES fueron vigentes desde comienzos del presente milenio hasta el 2009, para ser aplicados a estudiantes de último año, como instrumento tendientes a medir la calidad de la educación, con contenidos específicos de cada una de las profesiones y disciplinas. A partir de ese momento, dentro del espíritu de la Ley 1324 y el Decreto 3963 de 2009, se denomina Examen de Estado de Calidad de la Educación Superior SABER PRO o Pruebas Pro (ECAES) el cual es un instrumento estandarizado para evaluar competencias transversales en estudiantes de todos los programas: escritura, razonamiento cuantitativo, lectura crítica, competencias ciudadanas e inglés. En cuanto a lo específico de la formación en salud, se miden competencias tales como: ética y bioética, salud pública y administración y gestión en salud. El examen se encuentra en período de construcción y el ICFES tiene previsto aplicar el diseño definitivo en Noviembre de 2012 (Google, Pruebas PRO (ECAES), Mayo 20 de 2012).
} 
sidades y en el foro de la Asociación de Facultades de Terapia Ocupacional (ASOCOLFACTO). En el momento, aproximadamente cinco (50\%) universidades con planes curriculares de Terapia Ocupacional participan activamente en el proceso liderado por el CNA, en las fases de autoevaluación, acreditación y re acreditación. Se esperaría que las universidades restantes se incorporen formalmente a la cultura de la calidad y asuman decididamente la evaluación y el mejoramiento continuo de los programas de formación. Y en cuanto a las Pruebas PRO, si bien es útil el diseño de exámenes genéricos por áreas, como por ejemplo Ciencias de la Salud, también es deseable que se diseñen mediciones de las competencias profesionales específicas, como sucedía con los ECAES, de manera que los resultados puedan ser utilizados por cada programa curricular con fines evaluativos de su propio proceso de estudios, tal como sucede en los Estados Unidos y Canadá, entre otros países.

Por otra parte, un aspecto de gran importancia y claramente deficitario en el contexto educativo es la inexistencia de oferta postgraduada (especialización, maestría, doctorado) en el campo de Terapia Ocupacional y/o de Ciencia Ocupacional/ Ciencia de la Ocupación Humana, como se identificó en el segmento sobre cuerpo de conocimientos. Este hecho se torna más preocupante si se piensa que la profesión tiene una trayectoria de 45 años en el país, lapso suficiente para el surgimiento de alternativas de postgrados en el terreno propio de la Terapia Ocupacional. El ofrecimiento de planes postgraduados es un factor altamente estratégico para la presencia nacional e internacional y la identidad fortalecida de la Terapia Ocupacional colombiana. Recordemos que en esos ambientes educativos se activan grupos de investigación tendientes a impulsar la generación de conocimiento y/o la prueba de hipótesis, enfoques y metodologías efectivas y novedosas. $Y$ por ese conducto contribuirían al fortalecimiento, utilidad y evaluación de la pertinencia social de los programas de atención y enriquecerían el intercambio y debates del grupo profesional.

\section{Especificidad y cobertura de los servicios humanos}

El fondo de conocimientos disponible y su expresión en planes académicos son aspectos fundamentales para los servicios humanos que llevan a cabo los profesionales. Puede decirse que es allí, en el contexto de la realidad, donde se pone a prueba el bagaje teórico-aplicado existente, su especificidad y efectividad, sus condiciones de oferta y demanda y por ende su pertinencia social y el grado en que la sociedad valora esos servicios. Aquí nos referiremos a dos aspectos centrales: la especificidad o singularidad de los servicios que se ofrecen en Terapia Ocupacional y su cobertura, producto de la oferta y la demanda.

La singularidad de la Terapia Ocupacional, está expresada claramente en su nombre: terapéutica de la ocupación y ocupación terapéutica. Esto implica reconocer el bienestar, autonomía y sentido de la realización humana inherente a las ocupaciones cotidianas que llevan a cabo las personas; entender cuándo el desempeño ocupacional se ve interferido por con- 
diciones adversas de orden biológico psicológico o social; y orientar la conservación o recuperación de las competencias ocupacionales, con el fin de que los seres humanos puedan mantener o recobrar su autonomía y sentido de bienestar de manera completa o parcial, en casos de limitaciones no reversibles.

Así pues, el conocimiento más central o nuclear de los terapeutas ocupacionales debe ser el de la ocupación en todas sus facetas y su experticia debe fundamentarse en el empleo de las ocupaciones habituales como instrumentos de atención efectiva. Cuando el ejercicio de un terapeuta ocupacional se enfoca principalmente en la ocupación, es decir cuando su práctica está basada en la ocupación y dirigida para alcanzar las metas y secuencias de desempeño esperadas para los consultantes de sus servicios, entonces puede decirse que está demostrando, con claridad y precisión, el valor de su actuación profesional.

El reto principal consiste en evaluar el repertorio de actividades socialmente pertinentes y significativas para cada persona, de manera que se genere su motivación y la movilización de sus recursos personales en la dirección esperada. Además, se debe atender, entre otros factores, a la percepción social de las ocupaciones, las condiciones socio políticas que las enmarcan, el grado de aceptación según edad, género, nivel educativo y grupo étnico y los antecedentes socioculturales y momento histórico que transforman las alternativas ocupacionales. Antes que tener datos cuantitativos sobre el uso de la ocupación terapéutica en el ejercicio profesional, lo que se conoce son apreciaciones frecuentes de colegas que reportan la disminución del empleo de ocupaciones como práctica terapéutica y la utilización de instrumentos comunes a otras profesiones, especialmente en los campos más tradicionales de las disfunciones físicas y la salud mental. Ante la insuficiente información sobre el asunto, pero dada su importancia para la identidad profesional, aquí hacemos un llamado a que la comunidad profesional diseñe formas que permitan determinar las características y frecuencia de aplicación de actividades terapéuticas en la práctica profesional, con miras a debatir el tema a profundidad y formular recomendaciones.

La cobertura de servicios, incluida Terapia Ocupacional, ha sido estudiada por la Superintendencia Nacional de Salud (1997, 2007 elaborado en 2001). El estudio de 1997 registra el comportamiento de cada profesión en una variedad de factores. Por ejemplo, en 1985 el número de terapeutas ocupacionales por 1000 habitantes en el país era 0.021 mientras que en el año 2000 la cifra se incrementó a 0.101 por 1000 habitantes (p. 79, Gráfico 8). Pero conocer el número de terapeutas ocupacionales por habitantes es solamente un dato. El estudio de 2001, efectuado por la Unión temporal Pontificia Universidad Javeriana CENDEXFedesarrollo-Family Health Foundation describe un panorama amplio del recurso de salud, las condiciones de oferta y demanda y sus proyecciones a 2020. Aunque la investigación presenta una restricción en cuanto que el comportamiento específico de Terapia Ocupacional no está desagregado, sino que aparece bajo el rubro "Terapias", sus resultados deben tenerse en cuenta, por ejemplo tres: 
(a) se precisa que, en salud, "Terapias" (Física, Fonoaudiología y Terapia Ocupacional) es el área de más alto crecimiento en oferta profesional ( $p$. 70), lo cual genera una brecha entre la cantidad de profesionales disponibles y las oportunidades de trabajo; (b) la oferta educativa (perfil curricular) en "Terapias" no se correlaciona con el mercado de servicios (perfil ocupacional), lo que significa un desfase entre formación y trabajo, tanto para las instituciones estatales como para los operadores públicos y privados; y (c) en cuanto a empleo, a través de un análisis prospectivo el estudio encontró que mientras en el año 2000 las "Terapias" tenían una desocupación de $48 \%$, ésta se incrementaría a $66 \%$ en el año 2020 en comparación con un $21 \%$ para Medicina.

Los hallazgos mencionados son preocupantes y hacen indispensable que las universidades, encargadas de la formación, y las agremiaciones profesionales, interesadas en la comunidad profesional, creen alianzas para estudiar a fondo los temas de oferta y demanda y la correlación entre perfiles de formación y perfiles ocupacionales, no sólo en el sector de la salud sino en educación y trabajo y puedan establecer vínculos con las instancias apropiadas en esos sectores a fin de diseñar las estrategias que se consideren pertinentes, como resultado de las reflexiones.

\section{Presencia gremial}

La importancia de crear nexos de carácter gremial, nacional e internacional, fue clara para las terapeutas ocupacionales que estuvieron a cargo del primer programa curricular en la Universidad
Nacional, a partir de 1966. Al producirse la creación de la Carrera de Terapia Ocupacional en la Universidad Escuela Colombiana de Rehabilitación en 1969, se compartieron metas comunes con las representantes de dicho programa, alcanzándose la creación de la Asociación Colombiana de Terapia Ocupacional (ACTO) en Junio 5 de 1972 y su vinculación a la World Federation of Occupational Therapists en 1974, previo cumplimiento de los requisitos para la aprobación de los dos programas académicos, por esa entidad.

A partir de los años setenta del anterior milenio, la ACTO ha tenido una presencia permanente, no exenta de dificultades de diversa índole, una de las cuales ha sido el bajo número de profesionales vinculados, porque es innegable que a mayor cantidad de asociados mayor probabilidad de cumplir metas comunes y alcanzar creciente fortaleza asociativa, o a la inversa. No obstante las dificultades, la ACTO ha logrado permanecer vigente, gracias a la dedicación de un grupo de colegas que acepta comprometer parte de su tiempo, en calidad ad honorem, para impulsar el cumplimiento de los objetivos y funciones establecidos en los Estatutos y constituirse en voceros de las causas de la profesión como totalidad. Es así como se han fortalecido los nexos e intercambios con la Federación Mundial de Terapeutas Ocupacionales. La ACTO contribuyó a la iniciación de la Confederación Latinoamericana de Terapia Ocupacional en 1997 cuando, en Caracas, se produjo su creación; allí Colombia obtuvo la Vicepresidencia (Trujillo, 2002, p. 536). En 2001 se creó la Asociación Colombiana de Facultades de Terapia Ocupacional (Junta Direc- 
tiva, CCTO, 2012) y en 2011 la Asociación fue transformada en Colegio Colombiano de Terapia Ocupacional (CCTO). Este paso, que fortalece a la agremiación, fue precedido de un estudio cuidadoso por parte de la Junta Directiva y la consulta a expertos y a la Asamblea General, a fin de ajustarse a la Ley 1164 de 2007 que establece disposiciones relacionadas con la planeación, formación, vigilancia y control del ejercicio, desempeño y ética del talento humano en salud (Junta Directiva ACTO, 2011).

El CCTO enfrenta varios desafíos importantes, uno de los cuales es alcanzar la vinculación de la mayoría de terapeutas ocupacionales que ejercen en Colombia. Para alcanzar ese punto, se requiere que la colectividad perciba la presencia del Colegio como indispensable en la supervivencia y progreso de la Terapia Ocupacional. $Y$ de hecho su existencia es fundamental, en alto grado. A manera de ejercicio para la escritura del presente artículo, la autora procedió a reflexionar sobre logros del Colegio, a partir de los Objetivos y Funciones contenidos en el Capítulo II de sus Estatutos, encontrándose realizaciones en todos los órdenes. Solamente se ejemplifican algunas, a saber: desarrollo de Estándares Profesionales; preparación y divulgación del Código de Ética en varias versiones; elaboración y trámite de la Ley de Ejercicio Profesional y su actualización y renovación(1982, 1988, 2005); planeación y puesta en marcha de 16 Congresos de Terapia Ocupacional con invitados nacionales e internacionales; planeación, diseño y publicación de la Revista Ocupación Humana por veintitrés años consecutivos (1983-2006) y retorno en $2012^{7}$; elaboración de Boletines Informativos y comunicados de manera periódica, en físico y posteriormente en versión virtual; vocería permanente ante instancias gubernamentales, públicas y privadas; vínculos con entidades internacionales; promoción de la investigación entre los asociados; auspicio de múltiples jornadas y eventos para el fomento de la Terapia Ocupacional; y reflexiones y transformaciones sobre la imagen visual de la profesión.

El ejercicio anterior permite imaginar cuáles serían las consecuencias sustancialmente debilitantes para la Terapia Ocupacional, de no contar con la dedicación y logros obtenidos durante el proceso evolutivo ACTO... CCTO. Una estrategia más potente que el ejercicio descrito en el segmento previo podría ser que la Junta Directiva aceptara preparar un Reporte, con fines informativos y pedagógicos, dirigido a la totalidad del gremio que no ha estado cerca del trabajo realizado ni de los resultados producidos, en el cual se diera cuenta amplia sobre la manera como el CCTO ha contribuido a "fortalecer la consolidación de la Terapia Ocupacional como profesión" (Artículo 6, numeral 1, capítulo II, Estatutos del Colegio Colombiano de Terapia Ocupacional), lo que es análogo a decir que ha aportado significativamente al bienestar de todos los terapeutas ocupacionales en Colombia y que ese aporte tendría dimensiones exponenciales de contar con una membresía mayoritaria.

\footnotetext{
${ }^{7}$ Nota de la editora: Si bien el relanzamiento de la revista estaba previsto para 2012, debió postergarse para 2013 por diferentes razones; algunas de ellas se abordan en este artículo.
} 


\section{Mirada al futuro}

Los temas que se han presentado en los títulos Aspiraciones y Avances nos plantean horizontes que se pueden entender como conclusiones y recomendaciones y que se agrupan en cuatro categorías relacionadas con el fortalecimiento de la profesión así: fondo de saber específico; centralidad de la ocupación; agremiación profesional; y el terapeuta ocupacional como protagonista.

\section{Fondo de saber específico}

En el momento de desarrollo actual de la Terapia Ocupacional en Colombia, con la experiencia ganada en los campos académico, de servicios humanos directos e indirectos y de gestión gremial, con especificidades culturales para compartir y con múltiples oportunidades de intercambio asociadas a la globalización y a los avances tecnológicos, es necesario trazarse la meta de pasar a divulgar conocimientos y experiencias, así contribuir al crecimiento del fondo de información internacional $y$, ante todo, fortalecer el saber específico necesario para el país.

No es infrecuente el llamado de miembros de la comunidad profesional a emplear saber nacional y latinoamericano para sustentar el ejercicio. Al respecto puede decirse que, por una parte, hay postulados, principios, enfoques y metodologías que tienen un carácter universal y en esa medida es legítima su utilización o trans- ferencia, independientemente de la región en la cual se produzcan. Tal afirmación es válida si se comparte la premisa de que el saber es patrimonio universal. Por otra parte, se reconoce que algunas posturas filosóficas, conceptuales, aplicadas y experienciales están asociadas a la historia y realidad socioeconómica, política, cultural y geográfica. En ese sentido, es nuestra responsabilidad dar respuesta a esas especificidades a través de la producción y divulgación de información y conocimiento local o regionalmente orientado.

Una estrategia potente para alcanzar un desarrollo más acorde con la meta de generar conocimiento, culturalmente enfocado, consiste en promover la formación postgraduada de un porcentaje mayoritario de terapeutas ocupacionales, a través del ofrecimiento de programas de maestría en el corto plazo y de doctorado en el mediano y largo plazo ${ }^{8}$. Esta empresa podría estar liderada inicialmente por las universidades que se encuentran más avanzadas en el proceso de autoevaluar su calidad y cuyo programa universitario básico se encuentre acreditado por el Consejo Nacional de Acreditación y el Ministerio de Educación Nacional. El ofrecimiento de postgrados en el dominio de estudio de la Terapia Ocupacional y también centrados en el concepto fundamental que la sustenta, la ocupación humana, como es el caso de la Ciencia Ocupacional (Grupo Ocupación y Realización Humana, 2011), deberían constituir una alta prioridad por rela-

\footnotetext{
${ }^{8}$ La tendencia mundial en Terapia Ocupacional es la de ofrecer crecientemente programas educativos postgraduados, especialmente maestrías y doctorados. Algunos ejemplos son Australia, Brasil, Canadá, Chile, Estados Unidos, Gran Bretaña, Japón, México y Suecia.
} 
cionarse con los conceptos esenciales de la profesión.

\section{Centralidad de la ocupación}

La ocupación es el eje nuclear de la Terapia Ocupacional, lo cual significa que el fondo de saber y los esfuerzos investigativos deben orientarse a una prioridad: dar cuenta de y explicar la actuación ocupacional humana. En este campo singular los profesionales de la Terapia Ocupacional deben ser expertos para poder colaborar de manera crecientemente efectiva con las personas que necesitan sus servicios y ser reconocida su calidad por la sociedad. Allí radica la especificidad; en todas las facetas de por qué la ocupación es esencial para los seres humanos y de qué manera el involucrarse en ocupaciones permite la recuperación del bienestar y cómo a través de la ocupación se puede restaurar la calidad de vida. El instrumento y mayor valor agregado de los terapeutas ocupacionales es la comprensión y manejo de la ocupación terapéutica en su contexto personal y sociocultural.

Debe señalarse, sin embargo, que las ocupaciones específicas no son estáticas, como tampoco el valor que se les asigna en un período histórico determinado. Por eso, un gran reto que deben resolver los terapeutas ocupacionales es comprender que la ocupación y los repertorios ocupacionales se transforman con el paso del tiempo, lo cual obliga a identificar y analizar las formas ocupacionales personal y socialmente prevalecientes y comprender los por qué y cómo de los cambios, a fin de orientar a las personas en las ocupaciones cotidianas que sean más estimulantes y apropiadas en términos de conservar o recuperar el bienestar y avanzar en sus metas de vida, en el sentido de la realización humana.

Dado el carácter estratégico de la ocupación para el ejercicio de Terapia Ocupacional y sin disponer de estudios que hayan profundizado sobre las ocupaciones habituales en la cultura colombiana y sobre sus tendencias en la práctica profesional, se propone crear una comisión de alto nivel, integrada por representantes de los programas curriculares de Terapia Ocupacional, por terapeutas con práctica profesional en diversas áreas del ejercicio y por delegados del CCTO. La meta consistiría en elaborar un conjunto de proposiciones que orienten a la comunidad profesional sobre concepciones y comprensión de ocupaciones y establecer criterios para la investigación y utilización de la ocupación en el ámbito curricular y de la prestación de servicios humanos. El propósito sería fortalecer la personalidad e identidad ocupacional, los servicios humanos y el reconocimiento social.

\section{Agremiación profesional}

Los propósitos asociativos, que cumplen las agremiaciones, son indispensables en el mundo contemporáneo cuando se trata de fortalecer causas comunes. A lo largo de la historia de las civilizaciones y muy claramente en la actualidad, se demuestra cuan potente es aunar esfuerzos en búsqueda del bien colectivo y la importancia de los resultados que se alcanzan al reunir cualidades y talentos individuales. Más aún, en la realidad que vivimos, dada la magnitud y celeridad de los asuntos que se deben resolver, resulta 
estratégico establecer alianzas y convenios de cooperación entre asociaciones con diferentes énfasis sociales. A mayor madurez de los grupos humanos, mayor fortaleza de sus agremiaciones para ofrecer a los asociados oportunidades y servicios que se reflejen en bienestar individual y de la causa que se apoya.

El CCTO tiene por delante muchos retos, algunos de los cuales son motivar la vinculación de nuevos asociados; promover la construcción de una comunidad profesional participativa y deliberante; avanzar en credibilidad ante organismos e interlocutores de los niveles político, empresarial y social amplio; e incrementar sus relaciones de cooperación nacional e internacional, para promover "el desarrollo científico, investigativo, ético y normativo de la profesión" (Objetivo 2, Capítulo II, Estatutos Colegio Colombiano de Terapia Ocupacional). Tenemos confianza en que cumplirá sus propósitos de acuerdo a los objetivos y funciones consignados en los Estatutos. En el caso del presente artículo, se propone una ruta a futuro, derivada de la discusión expuesta y cuya puesta en marcha involucra el establecimiento de alianzas con ACOLFACTO y con universidades específicas que ofrezcan programas de Terapia Ocupacional.

\section{Construcción de bases de datos}

Las bases de datos o conjunto de información relacionada, agrupada y almacenada se conciben como el cerebro de las organizaciones porque permiten que las personas y colectivos tomen decisiones de manera sustentada. En Terapia Ocupacional necesitamos disponer de manera ágil y oportuna de datos válidos y confiables que puedan ser correlacionados de diferentes formas y así permitan visualizar panoramas de interés profesional, para efectos de entender diversas situaciones y adoptar medidas. Por ejemplo, se necesita disponer de un censo o caracterización amplia y actualizada sobre quiénes son los terapeutas ocupacionales en el país, su distribución geográfica, género, rango de edades, universidad y año de grado, tipo de vinculación profesional, área(s) de experticia, procedimientos de intervención que emplean, antigüedad, salarios, publicaciones y percepción de la Terapia Ocupacional por parte de diversos actores sociales, entre otros datos.

Construir una base de datos técnicamente diseñada, actualizada, efectiva y útil para la toma de decisiones es una empresa colectiva en la que deberían participar varios expertos y organizaciones, por concurso y en alianza con ACOLFACTO y con universidades individuales, las cuales también se benefician al disponer de información válida y confiable sobre el comportamiento del ejercicio profesional en el país, con fines de planeación, puesta en marcha de proyectos y autoevaluación. Algunos análisis que podrían emprender el Colegio y las Universidades son los de oferta y demanda local, regional y nacional, distribución por áreas de ejercicio, oportunidades de empleo, receptividad social a la Terapia Ocupacional, utilidad y reconocimiento por áreas de ejercicio, entre otros. Fuentes para divulgar aspectos de la información serían la página web del CCTO, que se vería significativamente enriquecida, la Revista Ocupación Humana y los resultados de infor- 
mes investigativos de grupos o autores individuales. $\mathrm{Y}$ ante todo, con mayores herramientas para monitorear la realidad, se estaría velando por el desarrollo de la Terapia Ocupacional en Colombia.

\section{El terapeuta ocupacional como protagonista}

Los terapeutas ocupacionales son quienes construyen la historia de su profesión y de las instituciones que la soportan. Sobre los comportamientos que deben demostrar, se han pronunciado tanto las universidades, a través de perfiles de estudiantes, como el CCTO por medio del Código de Ética para el ejercicio. Aun así, conviene expresar aquí algunas ideas sobre el tema de cualidades profesionales, personales y ciudadanas, al reconocer que los terapeutas ocupacionales actúan en el campo de los servicios humanos, que su profesión combina principios científicos, humanistas y de sensibilidad social y que las sociedades actuales atraviesan épocas de turbulencia y crisis.

A nuestro entender el deber ser del terapeuta ocupacional colombiano ha de consistir en un conjunto de cualidades como las siguientes:

1. Actuación dirigida al bienestar de las personas atendidas y a la mayor visibilidad social de la profesión, a partir de un desempeño individual e institucional con identidad ocupacional, alta calidad y pertinencia social.

2. Iniciativa y capacidad de interacción para establecer diálogos interprofesionales y expandir vínculos y alianzas estratégicas, locales, regionales, nacionales e internacionales, en los niveles de su competencia.
3. Participación efectiva y liderazgo en asociaciones profesionales con el fin de asegurar su fortalecimiento, en beneficio de la colectividad y de la sociedad.

4. Desarrollo de capacidades y habilidades para comunicar la imagen y los beneficios de la Terapia Ocupacional ante instancias y representantes responsables de generar política local, regional y nacional.

5. Comprensión y actuación dirigida por postulados de derechos y deberes ciudadanos, sentido de la justicia, convivencia respetuosa, reconocimiento de la diversidad humana, aceptación de normas y limites sociales, solución racional y argumentada de conflictos y auténtico interés por contribuir al bien colectivo.

En conclusión, los terapeutas ocupacionales deben dar pruebas de una actuación que correlacione: principios éticos; formación académica sólida y actualización permanente; ejercicio con identidad y pertinencia social que se traduzca en visibilidad creciente de la Terapia Ocupacional; vinculación gremial efectiva; y manejo experto de los factores político-económicos y socioculturales que influyen en la historia ocupacional de las personas y en el desarrollo de la profesión.

\section{Referencias}

Álvarez, L. (2010). Terapia ocupacional. Un enfoque sensorial en la escuela. Bogotá: Editorial Marpe Publicidad.

Etzioni, A. (Ed). (1969). The semiprofessions and their organization: Teachers, nurses and social workers. New York: Free Press.

Grupo Ocupación y Realización Humana (2011). Ocupación: sentido, realización y libertad. Bogotá: Editorial Universidad Nacional de Colombia. 
Junta Directiva Asociación Colombiana de Terapia Ocupacional (2011). Carta abierta a la comunidad de terapeutas ocupacionales. Bogotá: Enero.

Ministerio de la Protección Social en colaboración con Pontificia Universidad Javeriana CENDEX - Fedesarrollo - Family Health Foundation (2007). Segunda Edición. Recursos humanos de la salud en Colombia. Balance, competencias y prospectiva. Bogotá: Ministerio de la Protección Social.

Palm, R. del C.M., Navas, A., Fernández, A., Puche, A.R., Navarrete S., Mengelberg, E.G. y Bolaños, M.C. (2012). Catálogo Latinoamericano de Libros y Revistas de Terapia Ocupacional. Curitiba, Brasil.

Presidencia, Colegio Colombiano de Terapia Ocupacional (2012). Historia de la Asociación Colombiana de Terapia Ocupacional. Bogotá.

Sánchez, M. y Sáez, J. (2012) El estudio de las profesiones: la potencialidad del concepto de profesionalización. Monografía. Google, Mayo 8.

Superintendencia Nacional de Salud (1997). El talento humano de la salud en Colom- bia. Segunda Edición. Bogotá: Ministerio de Salud.

Trujillo R., A. (2009a). El terapeuta ocupacional que requiere el país: formación y actuación profesional. Bogotá: Universidad Colegio Mayor de Nuestra Señora del Rosario.

Trujillo R., A. (2009b). El presente y el futuro del terapeuta ocupacional. Bogotá: Facultad de Medicina, Universidad Nacional de Colombia.

Trujillo R., A. (2007). Cuarenta años de la terapia ocupacional en la Universidad Nacional de Colombia. Avances, aprendizajes y retos. Bogotá: Auditorio Alfonso López Pumarejo, Universidad Nacional de Colombia.

Trujillo R., A. (2002). Terapia ocupacional. Conocimiento y práctica en Colombia. Capítulo 7. Comunidad profesional. Bogotá: UNIBIBLOS.

Villamil F., O. L., Mojica, J., Puerto, A. Primera Ed. (2006). Terapia ocupacional en comunidad. Bogotá: Universidad Manuela Beltrán. 